\title{
The climate issue
}

\author{
Quantitative estimates presented in this issue demonstrate that astronomers contribute more to climate change \\ than the average global citizen. Concerted actions are needed to reduce the ecological impacts of our occupation.
}

life he world is in the midst of a global crisis that threatens our way of life, our livelihoods and in some cases, our actual lives. It is a harrowing sign of the times that at least two subjects fit that description. In this case, it is not the COVID-19 pandemic that is the topic of this issue of Nature Astronomy, but the climate emergency. The planet is warming, as indicated by tracking ocean heat and measuring temperatures in the different levels of the Earth's atmosphere. Global warming, the key manifestation of climate change, is linked to increased levels of greenhouse gases, such as carbon dioxide, and there is strong evidence that the rise of these gases in the atmosphere is anthropogenic. The planet has already warmed an average of $1{ }^{\circ} \mathrm{C}$ since the end of the nineteenth century and a further rise of that magnitude would have disastrous consequences for ecosystems. Given the connection between our actions and the habitability of our planet, we should examine whether our behaviours as individuals are worsening climate change.

This month's issue features a Perspective by Adam Stevens and colleagues that looks in detail at the work-related greenhouse gas emissions of the Australian astrophysics community. It serves not only to educate, but also to stimulate similar examinations in astronomical institutes across the globe. The authors quantify the equivalent number of tonnes of $\mathrm{CO}_{2}\left(\mathrm{tCO}_{2} \mathrm{e}\right)$ generated by the typical activities of our profession: operating observatories, using supercomputers, travelling to conferences and meetings. The average Australian astronomer generates at least $37 \mathrm{tCO}_{2} \mathrm{e}$ per year, on top of personal emissions. This figure is $40 \%$ higher than that of the typical Australian adult, and five times greater than the global average. Surprisingly, the majority of emissions

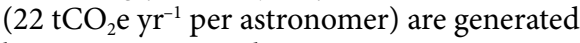
by operating several supercomputing centres used by astronomers. Coming only a distant second in the ranking, flight-related emissions make up another $6.1 \mathrm{tCO}_{2}{\mathrm{e} \mathrm{yr}^{-1}}^{-1}$ per Australian astronomer. This figure is unexpectedly low, given that international travel from Australia usually involves a

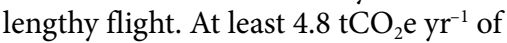

emissions per astronomer are generated by the operation of Australian observatories, which rely on a combination of grid, local solar power and diesel for electricity. The remainder of the assessed emissions (3.2 $\mathrm{tCO}_{2} \mathrm{e} \mathrm{yr}^{-1}$ per astronomer) are due to campus operations: lighting, heating and local computer usage.

For some context, in a Comment Knud Jahnke and colleagues have performed a similar analysis for their home institute in Europe: the Max Planck Institute for Astronomy (MPIA) in Heidelberg. They calculate a much smaller amount of emissions from work-related activities:

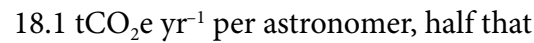
of an Australian, and the breakdown is also different. For MPIA, air travel dominates the inventory $\left(8.5 \mathrm{tCO}_{2} \mathrm{e} \mathrm{yr}^{-1}\right)$, followed by electricity - including supercomputing usage - at $5.2 \mathrm{tCO}_{2} \mathrm{e} \mathrm{yr}^{-1}$. Part of the difference is due to the method of calculating the impact of flight emissions (an important consideration, given that adjusting for this difference largely reconciles the flight-related components of the two estimates). The vast difference between electricity/supercomputer values mostly reflects Australia's usage of fossil fuels compared to Germany.

MPIA operates no observatory, and for some comparison on that front, Nicolas Flagey and co-workers have totalled the emissions for the 2019 operation of the Canada-France-Hawaii Telescope (CFHT) on Mauna Kea in another Comment. They

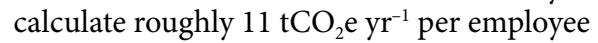
for telescope operations alone, and 16.5 $\mathrm{tCO}_{2} \mathrm{e} \mathrm{yr}^{-1}$ per employee for the observatory as a whole (including flights, vehicles, offices and housing) - apparently more expensive than the Australian telescopes, but almost the same as an MPIA researcher.

A large part of Australian and MPIA emissions are generated by supercomputers, to a degree that depends on the source of their electricity. Turning to renewable energy sources seems like a more sensible idea than reducing supercomputer usage, but Simon Portegies Zwart also supports another strategy in his Comment: become a more proficient programmer. Running Python on a single CPU core of a powerful workstation has a relatively high environmental cost; instead, prefer $\mathrm{C}++-$ it is faster anyway.

Air travel evidently has a major environmental impact. Conferencing is a large reason for astronomers to fly, but a quantitative comparison between a face-to-face and a virtual European Astronomical Society meeting by Leonard Burtscher and co-authors in their Comment shows that our traditional way of doing conferences produces at least three orders of magnitude more emissions than an online alternative.

Astronomers, then, contribute disproportionately to the hastening of climate change through the nature of our occupation. Climate change will not only harm ecosystems on our planet, it will also harm the observational nature of our work: Faustine Cantalloube and colleagues succinctly outline the detrimental effects of climate change on astronomy in their Comment. There are individual restitutional actions that we can take, from quantifying and analysing our emissions in the detailed manner of Stevens et al. to carbon offsetting and thinking twice before we execute a code or book a flight to a far-flung conference. As members of institutes and organizations, we can advocate for more environmentally friendly policies and practices. Ultimately, though, any individual actions we take will pale in comparison to corporate and industrial pollution. Astronomers have an "ethical obligation ... that must not be ignored", as Stevens et al. say, but we should not internalize environmental guilt; instead, we must call for systemic change and fight against bad practice; we must not let those governments eschewing their responsibilities (primarily China, the United States and India) escape from the spotlight; we must vote wisely and spend our money judiciously. Our planet is under threat; if astronomers care as much about habitable planets as the media think we do, we should start with the one under our feet.

Published online: 10 September 2020 https://doi.org/10.1038/s41550-020-01216-9 